\title{
Incorporating ecological context: a revised protocol for the preservation of Nepenthes pitcher plant specimens (Nepenthaceae)
}

\author{
C. Clarke ${ }^{1}$, J.A. Moran ${ }^{2}$
}

\section{Key words}

ecology

herbarium specimens

Nepenthes

taxonomy

\begin{abstract}
Pitcher plants of the family Nepenthaceae are vines or subscandent shrubs which produce modified lea organs that in most species serve to attract, trap, retain and digest animals for nutritional benefit. The sole genus within the family, Nepenthes, is abundant and diverse in Malesia. Previous taxonomic treatments of Nepenthes have relied almost entirely on the morphological features of the plants, with characteristics of the pitchers, inflorescences, leaf blades and indumentum being the most informative. Recent ecological research demonstrates that unique morphological characteristics and trap geometries provide useful taxonomic information, but this is often lost or obscured when specimens are prepared for herbaria by pressing them. In this paper, we demonstrate the value of ecological information in distinguishing between controversial montane Bornean taxa and provide a revised protocol for the collection and preparation of Nepenthes specimens, which is designed to maximise the amount of ecological information retained in herbarium material.
\end{abstract}

Published on 5 October 2011

\section{INTRODUCTION}

The genus Nepenthes L. (Nepenthaceae) comprises approximately 120 species of vines or subscandent shrubs (Cheek \& Jebb 2001, Phillipps et al. 2009, McPherson 2009), the majority of which are confined to the Malesian phytogeographic region. Centres of diversity and endemism include Borneo, Sumatra and the southern Philippines (Cheek \& Jebb 2001). All Nepenthes are pitcher plants, producing highly-modified, jug-shaped leaves at the tips of tendrils that arise from the apices of the leaf blades (Jebb \& Cheek 1997). The pitchers serve primarily to attract, trap, retain and digest animals for nutritional benefit (Lloyd 1942, Clarke et al. 2009). The majority of Nepenthes demonstrate marked pitcher dimorphism: juvenile plants tend to produce ovoid pitchers that rest on the ground (and are known as 'lower' or 'terrestrial' pitchers), whereas mature plants produce narrower, funnel-shaped pitchers (called 'upper' or 'aerial' pitchers).

The status of several taxa in Nepenthes is controversial, with a number of recently described species being distinguished from others on the basis of apparently minor morphological characteristics, some of which are of uncertain stability (Clarke \& Kruger 2006, Cheek \& Jebb 2009, Catalano 2009). One factor that contributes to the controversy is Danser's (1928) decision to not recognise sub-specific taxa. His influential monograph provided students of the genus with a rigorous and clearly defined protocol for describing and distinguishing taxa at specific rank. Minor and/or unstable morphological characteristics, such as plant or pitcher size, and variations in colour, were considered insignificant. All major, subsequent taxonomic treatments of Nepenthes (Jebb \& Cheek 1997, Clarke 2001,

\footnotetext{
${ }^{1}$ School of Science, Monash University Sunway Campus, Jalan Lagoon Selatan, 46150 Bandar Sunway, Selangor, Malaysia;

corresponding author e-mail: charles.clarke@sci.monash.edu.my.

2 School of Environment and Sustainability, Royal Roads University, 2005 Sooke Road, Victoria, BC, V9B 5Y2, Canada.
}

Cheek \& Jebb 2001) followed Danser's practice, but in the last few years there has been a departure from this approach, with several new taxa being distinguished from others using morphological characteristics that Danser (1928) would have considered to be insignificant (Clarke 2006). In one respect, this is not surprising: Danser's monograph is 82 years old and our collective knowledge of Nepenthes has increased substantially over that time. However, current trends in describing and distinguishing new taxa reflect those of the late 1800s, which resulted in many taxa that were poorly described or defined, or were distinguished on the basis of minor and/or unstable morphological differences, and led to considerable confusion among taxonomists and horticulturists alike.

Nepenthes have broad horticultural appeal and a number of enthusiasts now travel extensively through the natural habitats of Nepenthes. Their observations have led to the discovery and description of new taxa (e.g., McPherson 2009, Mey 2009, Catalano 2009), which has significantly enhanced our knowledge of the genus. However, horticulturists and enthusiasts often view the taxonomic importance of certain morphological traits differently from taxonomists and this may give rise to multiple, competing interpretations for some taxa. New discoveries are still being published on a regular basis (Lee et al. 2009, McPherson 2009, Robinson et al. 2009), making it difficult to address the issue at present.

However, recent ecological research has demonstrated that some controversies can be resolved through more detailed field observations and careful preparation of herbarium material. This is possible because various morphological and geometric characteristics of Nepenthes pitchers play important roles in trap function or specialised methods of nutrient acquisition, and some of these roles have only recently been elucidated. For instance, in $N$. albomarginata T.Lobb ex Lindl., a unique pitcher characteristic, which takes the form of a dense, tomentose band that lines the pitcher orifice, enables this species to target termites as a major source of prey (Moran et al. 2001, Merbach 
et al. 2002). Some other species appear to have evolved away from a strictly carnivorous mode of supplementary nutrition, with $N$. ampullaria Jack and $N$. lowii Hook.f. employing modified pitchers to trap significant amounts of leaf litter and tree shrew faeces, respectively (Moran et al. 2003, Clarke et al. 2009, Chin et al. 2010). Generally, less specialised species also appear to fall into three broad groupings:

1. Those that rely on a broad, expanded peristome (a ridge of hardened tissue that forms a 'collar' around the margins of the pitcher orifice) as the primary arthropod trapping mechanism. An example is N. bicalcarata Hook.f. (Bohn \& Federle 2004);

2. Those that rely more on a well-developed waxy zone on the inner surfaces of the pitchers, for example, N. gracilis Korth.; and

3. Those that employ a viscoelastic fluid in the pitchers as a prey retention mechanism, for example, $N$. inermis Danser, N. jacquelineae C.Clarke, Troy Davis \& Tamin, and N. rafflesiana Jack (which also utilises the two strategies outlined above (Clarke 2001, Gaume \& Forterre 2007)).

It is becoming apparent to ecologists that the degree of development of the peristome and waxy zone are fundamental to the prey-trapping strategies of many Nepenthes species (Gaume et al. 2002, Bohn \& Federle 2004, Gorb et al. 2004, Gaume \& Forterre 2007, Gaume \& Di Giusto 2009). This has important implications for taxonomists: if ecological characteristics can play a key role in distinguishing taxa, it is vital that as much ecological information is recorded and preserved in herbarium collections as possible.

Current 'best practice' for collecting herbarium specimens of Nepenthes involves collecting all parts of the plant: rosettes bearing lower pitchers, fragments of climbing stem bearing upper pitchers, and both and male and female inflorescences. Nepenthes specimens are difficult to press well, as the pitchers are often greatly distorted or damaged in the process. Furthermore, delicate structures such as the waxy zone on the inner surfaces of the pitchers (or the peristome itself) are particularly susceptible to damage through pressing, and important ecological information that can be derived through examination of the inner surfaces of the pitchers is often obscured in the process.

In this paper, we demonstrate how ecological research can be used to resolve taxonomic uncertainty about the status of a Bornean Nepenthes species, and propose revised 'best practice' methods for the collection of Nepenthes specimens for herbaria, so that the maximum amount of morphological and ecological information can be preserved.

\section{Nepenthes macrophylla (Marabini) Jebb \& Cheek - an example of a controversial taxon that is resolved through its ecological traits}

Nepenthes villosa Hook.f. is a spectacular montane Nepenthes species that is endemic to Mt Kinabalu and Mt Tambuyukon in northern Sabah, Borneo (Fig. 1a). Along with the giant N. rajah Hook.f., it generated substantial public and botanical interest when it was described in 1852 (see Phillipps et al. 2009), as the extraordinary degree of development of the peristome ribs was unlike that of any other pitcher plant known at that time. A few years later Hooker (1859) described a remarkably similar species from the same two mountains: Nepenthes edwardsiana H.Low ex Hook.f. (Fig. 1b). This species was distinguished from $N$. villosa by its elongated, tubular pitchers, simpler peristome structure, ebracteolate pedicels and sparser indumentum. Danser (1928) was of the opinion that these characteristics were insignificant, and reduced $N$. edward- siana to a synonym of $N$. villosa. In contrast, Harms (1936) reinstated $N$. edwardsiana.

More recently, Marabini (1987) described N. edwardsiana subsp. macrophylla, which is confined to the summit region of Mt Trusmadi, approximately $60 \mathrm{~km}$ SE of Mt Kinabalu. This taxon was distinguished from $N$. edwardsiana by its very large, ovate leaf blades and ovoid pitchers with broad, concave pitcher lids and less well-developed peristome teeth (Fig. 1c). Despite these marked differences, Marabini (1987) did not feel that they were significant enough to warrant distinction of the two taxa at specific rank. Jebb \& Cheek (1997) disagreed and raised $N$. edwardsiana subsp. macrophylla to specific rank, an interpretation that has been adopted by subsequent authors, but not without some reservations (see Clarke 1997).

On the basis of pitcher characteristics alone, the competing interpretations of $N$. macrophylla cannot be resolved objectively. However, Chin et al. (2010) demonstrated that N. macrophylla belongs to an extraordinary group of three Nepenthes species (the other two are N. rajah and N. lowii) that trap the faeces of mountain tree shrews (Tupaia montana Thomas (Scandentia)) for nutritional benefit. Tree shrews visit the pitchers to feed on nectar produced by glands on the pitcher lid, and the concave structure of the lid results in the nectar being accessible only if the tree shrews sit astride the pitcher (Fig. 1d). Tupaia montana marks the location of valuable resources with faeces, and as pitcher nectar appears to be an important food source, these animals frequently defecate into the pitchers (Clarke et al. 2009, Chin et al. 2010).

Chin et al. (2010) also demonstrated that the trap geometry of $N$. villosa is significantly different to that of $N$. macrophylla and that its pitchers do not trap tree shrew faeces. Field observations by $\mathrm{C}$. Clarke indicate that the same applies to $N$. edwardsiana. Clearly, T. montana distinguishes $N$. macrophylla from $N$. edwardsiana and $N$. villosa as a source of food, providing a compelling argument (in addition to the morphological characteristics listed by Jebb \& Cheek (1997)) for the recognition of the former taxon as a distinct species. While it is clear that field observations are essential to the elucidation of the relationship between $N$. macrophylla and tree shrews, it is likely that this association would have been detected much earlier if herbarium specimens included longitudinally dissected pitchers and brief notes about their contents, as this information is obscured when the pitchers are pressed.

\section{A 'best practice' method for the collection of Nepenthes specimens for herbaria}

Chin et al. (2010) demonstrated that characteristics such as pitcher orifice depth, lid reflexion angle and lid concavity are central to the faeces-trapping syndrome in N. lowii, N. macrophylla and N. rajah. Furthermore, it has become apparent to ecologists that both the development and extent of the wax zone inside the pitchers, and the microstructure of the inner surfaces of the peristome, can play important roles in prey capture (Bohn \& Federle 2004, Bauer et al. 2008). Unfortunately, the conversion of a markedly three-dimensional structure (such as a pitcher) to a two dimensional one by pressing herbarium material, causes much of this potentially valuable information to be lost. However, by making several modifications to the traditional method of collecting and pressing Nepenthes specimens, much of it can be retained and this should lead to more accurate and better informed interpretations of herbarium material. Accordingly, we propose the following protocol for the pressing and mounting of future collections of Nepenthes:

1. Collect stem fragments (separate ones if necessary) bearing both lower and upper pitchers (more than one pitcher of each type should be included in the collection); 

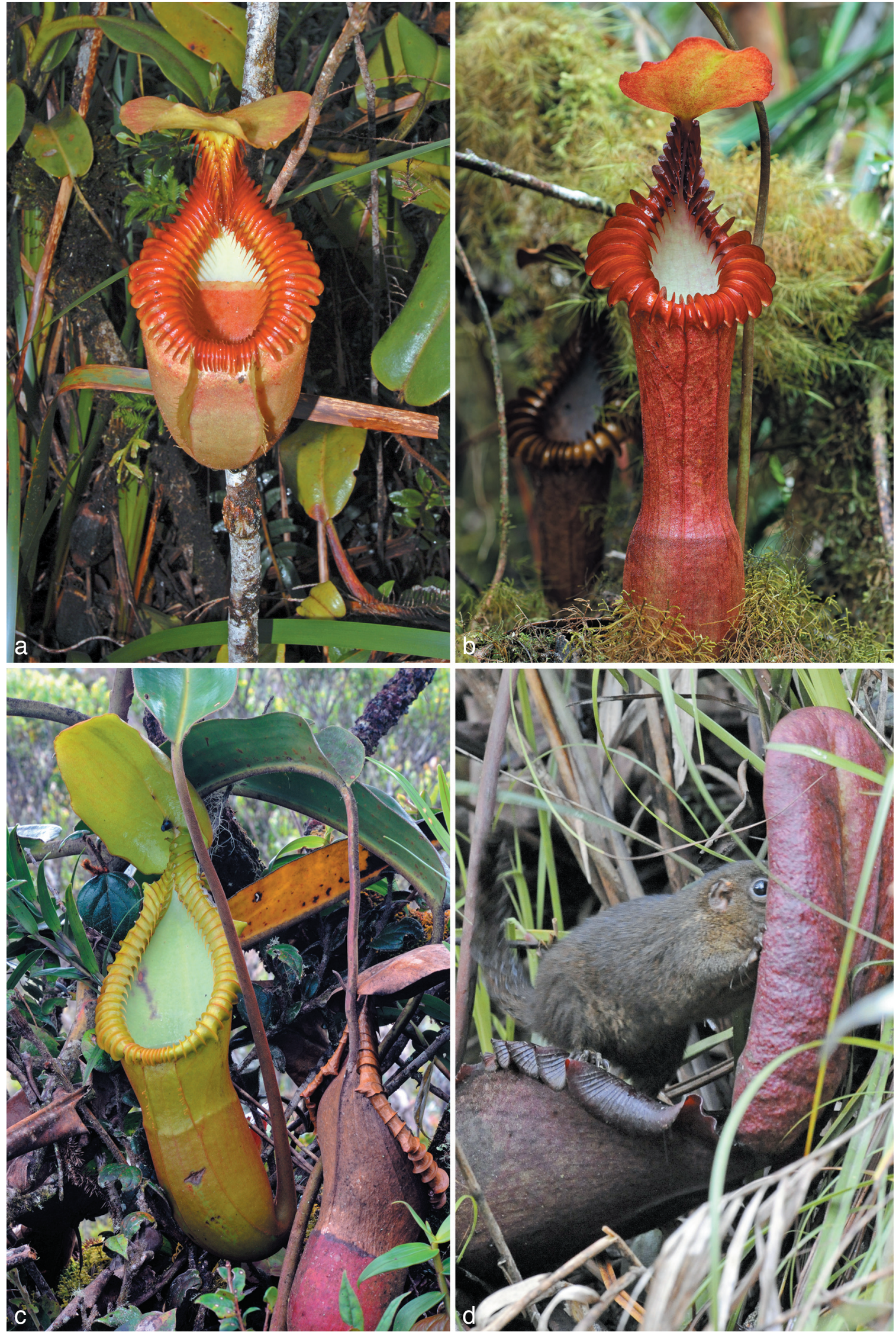

Fig. 1 Pitchers of a. N. villosa; b. N. edwardsiana; c. N. macrophylla; d. N. rajah; showing Tupaia montana feeding at the inner surface of the pitcher lid. - Photo a, c, d: C. Clarke; b: C.C. Lee.

2. Inflorescences of both sexes should be collected if possible and should not be torn from the stems, as the nature of attachment to the stem can provide useful taxonomic information;
3. At least one pitcher of each type should be dissected longitudinally from the midpoint at the front and rear of the pitcher and mounted so that the inner surfaces of both the pitcher and the peristome are facing outwards from the card; 
4. When pressing pitchers, try to preserve the angle of lid reflexion and concavity/convexity of the lids. If the lid angle cannot be faithfully preserved, indicate on the label what the approximate, typical angle of lid reflexion is, for both lower and upper pitchers. See Chin et al. (2010) for the measurement methodology;

4.1. Preservation of pitchers in alcohol can be an effective method of preserving trap geometry. Alternatively (and particularly for very large pitchers) a series of high-quality photographs that accurately depict trap geometry will serve a similar purpose.

5. Collection notes should include information about:

5.1. The habitat in which the material was collected (e.g. forest type, altitude, substrate);

5.2. Observations about conspicuous animal visitors to both the pitchers and the inflorescences (invertebrates and vertebrates);

5.3. Features unlikely to be obvious in preserved material (e.g. fragrance produced by pitchers (specify upper or lower form) or inflorescences (specify sex); viscosity of pitcher fluid (specify upper or lower form);

6. Deposition of material in the herbarium closest to the type locality. This makes it easier for field biologists (especially those based in Malesia) to study herbarium material economically and expeditiously; and

7. If possible, the contents of one upper and one lower pitcher should be extracted, dried and attached to the card in a clearly labelled, paper envelope.

\section{CONCLUSION}

Although it has been confirmed that N. rajah, N. lowii and N. macrophylla receive faecal inputs from tree shrews, several other species may also be candidates for this type of mutualism, but most of these are yet to be investigated because they grow in remote areas or herbarium material is scant. Three such species include N. ephippiata Danser and N. attenboroughii A.S.Rob., S.McPherson \& V.B.Heinrich, and N. truncata Macfarl. Given the challenges associated with studying these taxa in the field, ecologists are likely to study herbarium material as a precursor to field experiments. By following the protocol outlined above, the chances of obtaining useful background ecological information are substantially improved. For taxonomists, ecological information that can be related to morphological characteristics can assist in deciding upon the status of controversial taxa, as we have demonstrated here for $N$. macrophylla. To date, the nutrient acquisition strategies of less than $10 \%$ of Nepenthes species have been investigated. Our knowledge of the ecology of Nepenthes remains grossly deficient. Herbarium specimens that accurately preserve important ecological information can assist not only ecologists, but also taxonomists in their interpretations of the function of unique or atypical morphologies.

Acknowledgements We thank Ch'ien C. Lee for use of the photograph of N. edwardsiana.

\section{REFERENCES}

Bauer U, Bohn HF, Federle W. 2008. Harmless nectar source or deadly trap: Nepenthes pitchers are activated by rain, condensation and nectar. Proceedings of the Royal Society B 275: 259-265.

Bohn HF, Federle W. 2004. Insect aquaplaning: Nepenthes pitcher plants capture prey with the peristome, a fully wettable water-lubricated anisotropic surface. Proceedings of the National Academy of Science, USA 101: 14138-14143.

Catalano M. 2009. Nepenthes della Thailandia. Prague, Czech Republic.

Cheek M, Jebb M. 2001. Nepenthaceae. Flora Malesiana, Series I, Volume15.

Cheek M, Jebb M. 2009. Nepenthes group Montanae (Nepenthaceae) in Indo-China, with $\mathrm{N}$. thai and N. bokor described as new. Kew Bulletin 64: 319-325.

Chin L, Moran JA, Clarke C. 2010. Trap geometry in three giant montane Nepenthes species from Borneo is a function of tree shrew body size. New Phytologist 186: 461-470.

Clarke C, Kruger R. 2006. Nepenthes tenax C. Clarke \& R. Kruger (Nepenthaceae), a new species from Cape York Peninsula, Queensland. Austrobaileya 7, 2: 319-324.

Clarke CM. 1997. Nepenthes of Borneo. Natural History Publications, Kota Kinabalu, Sabah, Malaysia.

Clarke CM. 2001. Nepenthes of Sumatra and Peninsular Malaysia. Natural History Publications, Kota Kinabalu, Sabah, Malaysia.

Clarke CM. 2006. Introduction. In: Danser BH. 1928. The Nepenthaceae of the Netherlands Indies: 1-15. Natural History Publications (Borneo), Kota Kinabalu, Sabah, Malaysia.

Clarke CM, Bauer U, Lee CC, Tuen AA, Rembold K, Moran JA. 2009. Tree shrew lavatories: a novel nitrogen sequestration strategy in a tropical pitcher plant. Biology Letters 5: 632-635.

Danser BH. 1928. The Nepenthaceae of the Netherlands Indies. Bulletin du Jardin Botanique de Buitenzorg, Série III, 9: 249-438.

Gaume L, Di Giusto B. 2009. Adaptive significance and ontogenetic variability of the waxy zone in Nepenthes rafflesiana. Annals of Botany 104: 1281-1291.

Gaume L, Forterre Y. 2007. A viscoelastic deadly fluid in carnivorous pitcher plants. PLoS ONE 2: e1185.

Gaume L, Gorb S, Rowe N. 2002. Function of epidermal cells in the trapping efficiency of Nepenthes alata pitchers. New Phytolologist 156: 479-489.

Gorb E, Kastner V, Peressadko A, Arzt E, Gaume L, Rowe N, Gorb S. 2004. Structure and properties of the glandular surface in the digestive zone of the pitcher in the carnivorous plant Nepenthes ventrata and its role in insect trapping and retention. Journal of Experimental Biology 207: 2947-2963.

Harms H. 1936. Nepenthaceae. In: Engler A, Prantl K, Die natürlichen Pflanzenfamilien, 2 Aufl. Band 17b: 728-765.

Hooker JD. 1859. On the origin and the development of the pitchers of Nepenthes, with an account of some new Bornean plants of that genus. Transactions of the Linnaean Society, London 22: 415-424, t. 69-74.

Jebb M, Cheek M. 1997. A skeletal revision of Nepenthes (Nepenthaceae). Blumea 42: 1-106

Lee C, McPherson S, Bourke G, Mansur M. 2009. Nepenthes pitopangii (Nepenthaceae), a new species from central Sulawesi, Indonesia. Gardens' Bulletin Singapore 61, 1: 95-100.

Lloyd FE. 1942. The carnivorous plants. Chronica Botanica. New York.

Marabini J. 1987. Eine neue Unterart von Nepenthes edwardsiana Hook.fil. sowie Anmerkungen zur Taxonomie der Gattung Nepenthes L. Mitteilungen der Botanischen Staatssammlung München 23: 423-429.

McPherson SR. 2009. Pitcher plants of the Old World, Volume 1. Redfern Natural History Productions, Poole, UK.

Merbach MA, Merbach DJ, Maschwitz U, Booth WE, Fiala B, Zizka G. 2002. Mass march of termites into the deadly trap. Nature 415: 37.

Mey FS. 2009. Nepenthes bokorensis, a new species of Nepenthaceae from Cambodia. Carniflora Australis 7, 1: 6-15.

Moran JA, Clarke CM, Hawkins BJ. 2003. From carnivore to detritivore? Isotopic evidence for leaf litter utilization by the tropical pitcher plant Nepenthes ampullaria. International Journal of Plant Sciences 164: 635-639.

Moran JA, Merbach MA, Livingston NJ, Clarke CM, Booth WE. 2001. Termite prey specialization in the pitcher plant Nepenthes albomarginata - evidence from stable isotope analysis. Annals of Botany 88: 307-311.

Phillipps A, Lamb A, Lee CC. 2009. Pitcher plants of Borneo. 2nd edition. Natural History Publications, Kota Kinabalu, Sabah, Malaysia.

Robinson AS, Fleischmann AS, McPherson SR, Heinrich VB, Gironella EP, Peña CQ. 2009. A spectacular new species of Nepenthes L. (Nepenthaceae) pitcher plant from central Palawan, Philippines. Botanical Journal of the Linnaean Society 159, 2: 195-202. 